\title{
3D Printed Gelatin/Sodium Alginate Hydrogel Scaffolds Doped with Nano-Attapulgite for Bone Tissue Repair
}

\author{
Chun Liu' \\ Wen Qin' \\ Yan Wang ${ }^{2}$ \\ Jiayi $\mathrm{Ma}^{\mathrm{I}}$ \\ Jun Liu' \\ Siyu $\mathrm{Wu}^{\prime}$ \\ Hongbin Zhao' \\ 'Medical Research Centre, Changzhou \\ Second People's Hospital Affiliated to \\ Nanjing Medical University, Changzhou, \\ 213164, People's Republic of China; \\ ${ }^{2}$ Department of Clinical Laboratory, \\ Changzhou Second People's Hospital \\ Affiliated to Nanjing Medical University, \\ Changzhou, 213164, People's Republic of \\ China
}

Introduction: Bone tissue engineering (BTE) is a new strategy for bone defect repair, but the difficulties in the fabrication of scaffolds with personalized structures still limited their clinical applications. The rapid development in three-dimensional (3D) printing endows it capable of controlling the porous structures of scaffolds with high structural complexity and provides flexibility to meet specific needs of bone repair.

Methods: In this study, sodium alginate (SA)/gelatin (Gel) hydrogel scaffolds doped with different contents of nano-attapulgite were fabricated via 3D printing. The surface microstructure, hydrophilicity and mechanical properties were fully evaluated. Furthermore, mouse bone marrow-derived mesenchymal stem cells (BMSCs) were cultured with the composite hydrogels in vitro, and proliferation and osteoblastic differentiation were assessed. A rabbit tibia plateau defect model was used to evaluate the osteogenic potential of the composite hydrogel in vivo.

Results: When increasing nano-ATP content, the Gel/SA/nano-ATP composite hydrogels showed better mechanical property and printability. Moreover, Gel/SA/nano-ATP composite hydrogels showed excellent bioactivity, and a significant mineralization effect was observed on the surface after being incubated in simulated body fluid (SBF) for 14 days. The Gel/SA/ nano-ATP composite hydrogel also showed good biocompatibility and promoted the osteogenesis of BMSCs. Finally, histological analysis demonstrates that the Gel/SA/nano-ATP composite hydrogels could effectively enhance bone regeneration in vivo.

Conclusion: These properties render the Gel/SA/nano-ATP composite hydrogel scaffolds an ideal bone tissue engineering material for the repair of bone defects.

Keywords: 3D printing, attapulgite, composite hydrogel, osteogenesis, bone repair

\section{Introduction}

As some of the most common clinical conditions, bone defects are usually caused by severe fractures, osteoporosis, tumors, or infections, and the treatment of bone defects is still a great clinical challenge. ${ }^{1-3}$ Standard treatment strategies for bone defects employ autologous bone grafting, which has limited availability and may cause additional complications. ${ }^{4,5}$ Allografts, as another potential clinical option, are rarely used because of the potential risks such as disease transmission and immune rejection. ${ }^{6,7}$

Bone tissue engineering (BTE) materials for bone defect repair have gradually shown some satisfactory progress in the recent decades. ${ }^{8-12}$ The ideal bone repair materials should have good osteoinductivity or conductivity, better biocompatibility,
Correspondence: Hongbin Zhao Medical Research Centre, Changzhou Second People's Hospital Affiliated to

Nanjing Medical University, No. 68, Gehu

Road, Changzhou, Jiangsu, 213164,

People's Republic of China

$\mathrm{Tel} / \mathrm{Fax}+86$ I3919761032

Email zhao761032@I63.com 
sufficient mechanical strength, and appropriate degradation behavior. ${ }^{13,14}$ Three Dimensional (3D) printing is a rapidly developing technology that can be used for fabricating bioscaffolds. ${ }^{15,16}$ Bio-ink is dispensed onto a platform layer-bylayer to build a complex tissue construct with a 3D porous structure, controlled by a computer-aided design (CAD) model. Scaffolds prepared using 3D printing have many advantages, including controllable pore size and degradation rate, high structural complexity, and design flexibility. Thus, it has drawn wide attention in bone tissue engineering. ${ }^{17}$

Hydrogels are ideal materials for tissue engineering due to their good physicochemical properties, high water content (usually more than $90 \%$ ), capability of drug loading, ${ }^{18}$ excellent biocompatibility, and acceptable biodegradability. ${ }^{19-24}$ Their 3D hydrophilic cross-linked polymer networks could simulate the microstructure of the native extracellular matrix (ECM), providing a living microenvironment for cell growth. Numerous studies have shown that the hydrophilic properties of hydrogels may also affect the adhesion, proliferation, and differentiation of cells. ${ }^{25-29}$ Hydrogels crosslinked using natural polymers such as gelatin, hyaluronic acid, and fibroin have shown excellent biocompatibility; thus, the corporations have been widely used as scaffolds for growth factor transport and cell adhesion in BTE. ${ }^{30}$ In addition, some studies also showed that the peptide hydrogels are eminently suited as molecular scaffolds for bone and cartilage reconstruction. ${ }^{31}$ Sodium alginate (SA) has been extensively employed for bioprinting applications, and multivalent cationic transfer could induce its gelation. Moreover, it could also stably form cytocompatible hydrogels under physiological conditions due to its biocompatibility, biodegradability, and directional cell growth. ${ }^{32}$ However, alginate cannot provide mammalian cell-adhesive ligands, which limits cell adhesion. Numerous research results showed that gelatin (Gel) could improve cellular behavior. ${ }^{33}$ Gelatin is a biopolymer naturally derived from collagen with relatively low biotoxicity and acceptable degradation rate. ${ }^{34,35}$ According to recent reports, gels fabricated based on the combination of two or more different polymers via cross-linkers can tackle disadvantages and integrate advantages. ${ }^{36-39}$ Therefore, we tried to add gels to alginate to fabricate composite hydrogel scaffolds. Gelatin can be cross-linked with 1-ethyl-3-(3-dimethylaminopropyl) carbodiimide (EDC) and N-hydroxysuccinimide (NHS) solutions to achieve the double network cross-linking. However, the mechanical properties of the double network Gel/SA composite hydrogels are still weak and cannot meet the requirements of bone repair in BTE. Therefore, the development of high-strength hydrogels has been an emerging topic in the BTE field.
Attapulgite (ATP, $\left.\left(\mathrm{Al}_{2} \mathrm{Mg}_{2}\right) \mathrm{Si}_{8} \mathrm{O}_{20}(\mathrm{OH})_{2}\left(\mathrm{OH}_{2}\right)_{4} \cdot 4 \mathrm{H}_{2} \mathrm{O}\right)$ is a nanoscale hydrated layer-chain magnesium silicate mineral with rod-like crystalline morphology (Figure 1A), which possesses unique physicochemical properties, including high viscosity, large specific surface area, strong absorption ability, and abundant reserves in nature. ${ }^{40}$ In addition, nano-ATP has a special nanoscale structure in which nanorods can form a high-viscosity network structure resulting from the interactions among rods, which may promote osteogenesis when being doped in composite scaffolds according to the previous reports. ${ }^{28,41}$ Nano-ATP can also form a stable blend when combined with other polymer chains. These features render nano-ATP an appropriate material for osteoblast ingrowth. ${ }^{42,43}$ Nano-ATP rises up as a promising natural material in the chemical industry, plastics, and other industries in recent years. ${ }^{44-46}$ Novel applications of nano-ATP are still emerging, but their biomedical applications are rare to the best of our knowledge. Nano-ATP is non-toxic although it contains magnesium and aluminum in the form of $\mathrm{Al}_{2} \mathrm{O}_{3}$ $(9.88 \% \mathrm{Al}$ total). This amount is lower than that in kaolinite (39.5\%) and montmorillonite (16.54\%) which have been widely used in medicine. ${ }^{40}$ Nano-ATP was proved nontoxic in the early $1990 \mathrm{~s},{ }^{46,47}$ and several nano-ATP composite materials have been fabricated in our previous study, and in vitro and in vivo studies have confirmed the biocompatibility and the capability to promote osteogenesis. ${ }^{48,49}$

Here, Gel/SA/nano-ATP composite hydrogels containing different nano-ATP contents were fabricated via 3D printing. The biomechanical and physicochemical properties were characterized, and the printing parameters were optimized. Mouse bone marrow-derived mesenchymal stem cells (BMSCs) were cultured with the composite hydrogels, and proliferation and differentiation were assessed by in vitro assays. Finally, a rabbit tibia plateau defect model was used to evaluate the osteogenesis potential of the composite hydrogels. This article provides new ideas and preliminary results for designing and creating bone tissue repair scaffolds. It is also expected to provide new insights for the further development of nano-ATP composite hydrogels in the treatment of bone defects.

\section{Materials and Methods}

\section{Preparation of the Blend Hydrogel Inks}

Different ratios of blends were examined to prepare a composite hydrogel formulation for 3D printing. First, $10 \mathrm{~g}$ gelatin (Gel, $\sim 250 \mathrm{~g}$ Bloom, Aladdin Chemical Inc., Shanghai, China) was dissolved in $100 \mathrm{~mL}$ distilled water to 


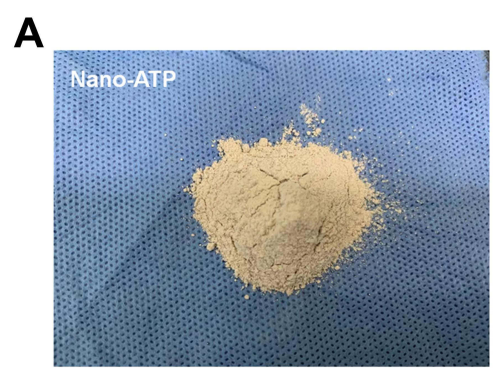

C

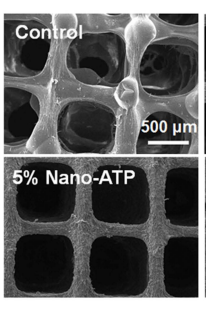

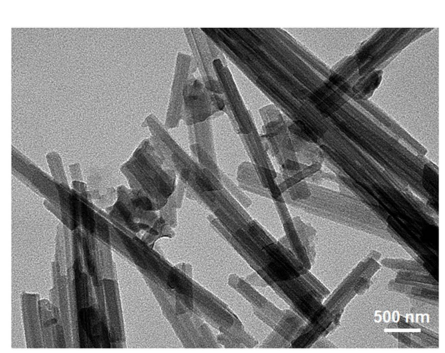

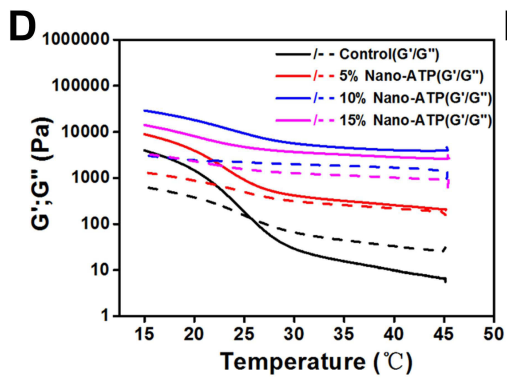

B

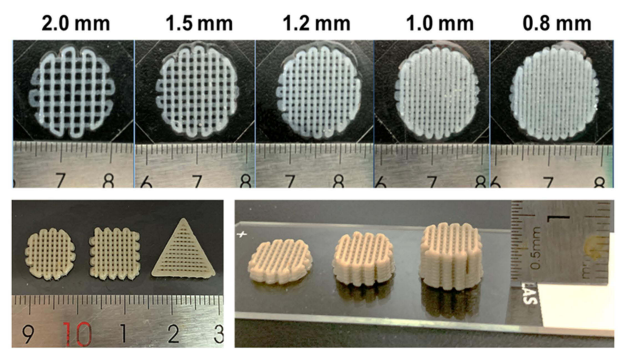

E

Figure I Morphology and rheological characterization of Gel/SA/nano-ATP composite hydrogel scaffolds with different nano-ATP contents. (A) Photograph and TEM image of nano-ATP powders with a rod-like crystalline morphology. (B) Representative digital photographs showing the printed structure with different grid spacing, shapes, and layers constructed from the composite hydrogel. (C) SEM images of composite hydrogel with different ratios of nano-ATP relative to the non-crosslinking composite hydrogel. (D) Rheological behavior of the composite hydrogel inks (viscosity and shear stress are plotted for shear rates of 0 to $100 \mathrm{~S}^{-1}$ ); (E) storage modulus ( $\mathrm{G}^{\prime}$ ) and loss modulus (G”) tested during cooling from 45 to $15^{\circ} \mathrm{C}$.

Abbreviations: Gel, gelatin; SA, sodium alginate; nano-ATP, nano-attapulgite; TEM, transmission electron microscopy; SEM, scanning electron microscopy.

obtain a uniform solution $(10 \mathrm{w} / \mathrm{v} \%)$ by stirring at a speed of $600 \mathrm{rpm}$ for $2 \mathrm{~h}$ at $50^{\circ} \mathrm{C}$. Sodium alginate (SA, $2 \mathrm{w} / \mathrm{v} \%$, Aladdin Chemical Inc., Shanghai, China) was then uniformly mixed into the gelatin solution. Different ratios of attapulgite powder (nano-ATP, 150-500 nm, Lanzhou Institute of Chemical Physics, Chinese Academy of Sciences, China) were then dispersed into the blend ( $0 \%$ (control), 5,10 , and $15 \mathrm{w} / \mathrm{v} \%$ ) solution to form the hydrogel inks.

\section{Rheological Analysis of Composite Hydrogel Inks}

Rheological characterization of the composite hydrogel inks was performed using an ARG2 rheometer (TA Instrument, USA). Briefly, the reaction solutions of the composite hydrogel blends were mixed in a syringe and quickly added on the plate of a rheometer. Small-amplitude oscillatory shear tests were performed by applying $5 \%$ strain amplitude with shear rates ranging from 0 to $100\left(\mathrm{~s}^{-1}\right)$ at $37^{\circ} \mathrm{C}$. The storage $\left(\mathrm{G}^{\prime}\right)$ and loss modulus $\left(G^{\prime \prime}\right)$ were recorded under temperature ramps. The temperature ramps were applied ranging from $15^{\circ} \mathrm{C}$ to $45^{\circ} \mathrm{C}$ with a constant rate of $1^{\circ} \mathrm{C} / \mathrm{min}$, the oscillation frequency and a constant shear amplitude were applied 1 $\mathrm{Hz}$ and $50 \mathrm{~Pa}$, respectively.

\section{D Printing of Gel/SA/Nano-ATP Composite Hydrogel Scaffolds}

The synthesis of the Gel/SA/nano-ATP composite hydrogel is shown in Scheme 1.

Specifically, the composite hydrogel bio-ink was loaded in a syringe linked to a $23 \mathrm{G}$ needle with a $0.33 \mathrm{~mm}$ inner diameter and installed in a screwassisted extrusion-based 3D bioprinter (regenHU, Villaz-St-Pierre, Switzerland). The syringe was heated at $37^{\circ} \mathrm{C}$ throughout the process due to the thermal gelation properties of Gel and the bio-ink was extruded when activating printing program. The composite hydrogel scaffold model was designed using CAD software (BioCAD, Villaz-St-Pierre, Switzerland). The scaffold has a $45^{\circ} / 135^{\circ}$ lay-down pattern, $300-\mu \mathrm{m}$ pore size and fiber diameter, and 4 layers of $2.88 \mathrm{~mm}$ height. The printing parameters included a extrusion pressure of $0.32 \mathrm{MPa}$ and a speed of $3 \mathrm{~mm} / \mathrm{s}$.

After printing, cross-linking of the composite hydrogel was achieved by being soaked in ionic solution for $4 \mathrm{~h}\left(\mathrm{CaCl}_{2}, 10 \mathrm{w} / \mathrm{v} \%\right.$, Aladdin $)$. The resulting hydrogels were then immersed in EDC and NHS (50 mM EDC and $25 \mathrm{mM}$ NHS in 95\% ethanol) solutions for crosslinking. Finally, the composite hydrogels were washed with deionized water to remove the residual EDC and NHS. 


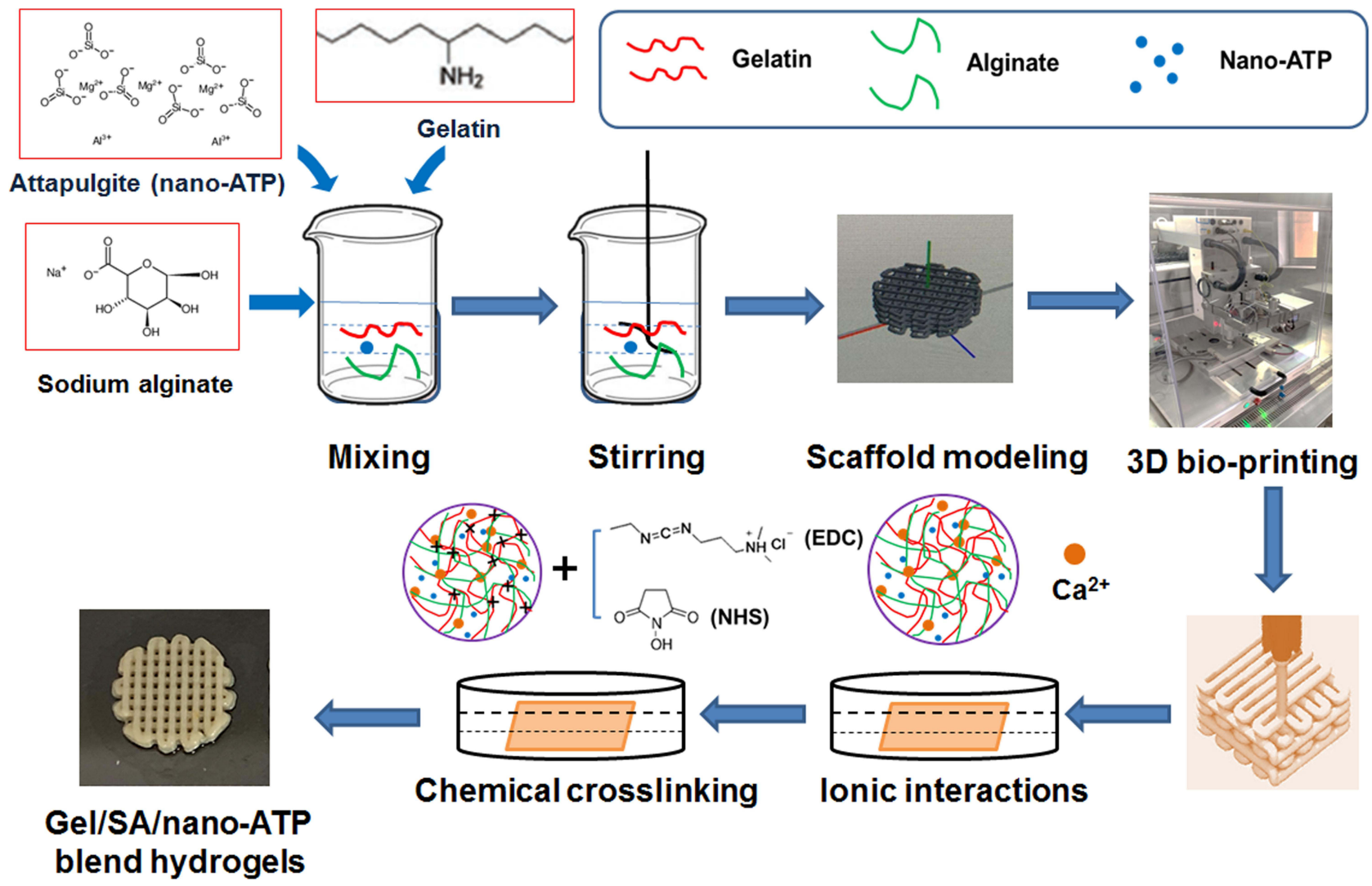

Scheme I Schematic illustration of the fabrication process of Gel/SA/nano-ATP composite hydrogel scaffold. Abbreviations: Gel, gelatin; SA, sodium alginate; nano-ATP, nano-attapulgite.

\section{Characterization of Composite Hydrogel Scaffolds}

All hydrogel samples were freeze-dried using a lyophilizer (Christ Alpha 1-2LD plus, Germany) and ground into a powder before characterization. The IR spectra of the Gel/SA/nano-ATP composite hydrogels were carried out by FTIR (Thermo Scientific Nicolet iS50, USA) at room temperature. Phase analysis of the composite hydrogel was performed by XRD (D/max2500 , Rigaku, Japan) with $2 \theta$ values of $5-50^{\circ}$ in a stepscan mode of $5^{\circ}$ per minute.

Thermogravimetry of the composite hydrogel was tested using a TGA analyzer instrument (TG 209 F3, Tarsus, Netzsch, Germany) with $10 \pm 0.1 \mathrm{mg}$ samples weighed into a platinum pan for thermal analysis and heated at $10^{\circ} \mathrm{C} / \mathrm{min}$.

Evaluation of the surface wettability of the composite hydrogel was conducted by a contact angle test instrument (FM4000, Krüss, Germany) at specific time intervals. The contact angle was recorded as the average of three measurements.

A Laser Zeta meter machine (Malvern Instruments, Zetasizer Nano ZSE) was utilized to determine the value of the zeta potential of the samples in water solution $(\mathrm{pH}=7.4)$.

\section{Mechanical Testing of Composite Hydrogel Scaffolds}

Samples used for the compression tests were measured using a uniaxial testing machine (Instron 5965, Pfungstadt, Germany). Composite hydrogels $(\mathrm{n}=4)$ were soaked in PBS solution $(\mathrm{pH}=7.4)$ for 1 day before the test comparing the freeze-dried hydrogels. The compression test was conducted until the samples were broken, and the stress-strain curves were obtained. Then the compressive strength was calculated according to the maximum stress before failure, and the linear range in the stress curve was used to calculate the compressive modulus.

\section{Microscopic Morphology Analysis of Composite Hydrogel Scaffolds}

The microstructure of the composite hydrogel was directly observed by a scanning electron microscopy instrument 
(SEM, JSM-6360LA, JEOL, Japan) on the surfaces and side sections. The sample surfaces were sputter-coated with an AuPd layer under an argon atmosphere before SEM observation.

\section{In vitro Mineralization of Composite Hydrogel Scaffolds}

SBF buffer simulates the content of different ionic species in human blood plasma as well as mineralization. The freeze-dried hydrogel samples were immersed in SBF (Yuanye Reagent Co., Ltd., Shanghai) and incubated for 14 days. After then, the samples were freeze-dried, and collected for further analysis of surface mineralization.

\section{Swelling of Composite Hydrogel Scaffolds}

The composite hydrogels were dried in an oven for at least 1 day and weighed $(\mathrm{Wd})$. The composite hydrogels were then swollen in PBS buffer $(1 \times, \mathrm{pH}=7.4$, Multisciences, Hangzhou, China) at $25^{\circ} \mathrm{C}$ and weighed every two hours (Ws) until the mass did not change obviously, after which equilibrium swelling was obtained. Experiments were carried out at least five times. The swelling was calculated according to the following formula. ${ }^{50}$

$$
\text { Swelling }(\%)=\frac{W s-W d}{W d} \times 100
$$

Here, Ws and Wd represent the weights of hydrogels under the swollen and dried state.

\section{In vitro Degradation Studies of Composite Hydrogel Scaffolds}

Degradation studies used gravimetric analysis. All freezedried weighed samples (W0) were swollen in PBS buffer at $37^{\circ} \mathrm{C}$ for $2,4,8$, or 12 weeks. At the selected time, the samples were freeze-dried and weighed (Wt). Experiments were carried out at least five times. The weight loss was calculated using Eq. (2). ${ }^{51}$

$$
\operatorname{Degradation}(\%)=\frac{W 0-W t}{W 0} \times 100
$$

Here, W0 and Wt represent the weights of the initial hydrogels and the weights at each time point.

\section{In vitro Biocompatibility and Osteogenesis Evaluations of Composite Hydrogel Scaffolds}

Mouse bone mesenchymal stem cells (BMSCs) were purchased from ATCC (ATCC, CRL-12424) and cultured in Dulbecco's-modified Eagle's medium (DMEM basic,
Gibco, ThermoFisher Biochemical Products, Beijing, China), which combined with $5 \% \mathrm{v} / \mathrm{v}$ fetal bovine serum (FBS, Biological Industries, Israel) and 1\% v/v penicillin/ streptomycin (Life Technologies Corporation, USA). BMSCs were cultured in an incubator with $5 \% \mathrm{CO}_{2}$ at $37^{\circ} \mathrm{C}$.

\section{CCK-8 Proliferation Assay}

The extract of the composite hydrogel was used to evaluate its compatibility co-cultured with cells by following the protocol of the Chinese National Standard (GB/T 169886.2017.12). Briefly, the composite hydrogel samples were sterilized with $75 \%$ ethanol for $12 \mathrm{~h}$ under UV light and then rinsed with PBS). Then the samples were immersed in DMEM basic medium with an extraction ratio of $0.1 \mathrm{~g} / \mathrm{mL}$. The supernatant was withdrawn and filtered through a $0.22 \mu \mathrm{m}$ membrane filter. Finally, 5\% $\mathrm{v} / \mathrm{v}$ FBS and $1 \% \mathrm{v} / \mathrm{v} \mathrm{P} / \mathrm{S}$ were added according to this proportion to produce a new cell culture medium.

Cell proliferation was measured via a CCK- 8 assay using the extracts described above. BMSCs were seeded onto a 96-well culture plate at a density of $5.0 \times 10^{3}$ cells/ well. After seeding for 1,3 , and 7 days, the culture medium in each well was removed, then $10 \mu \mathrm{L}$ of CCK8 buffer (Dojindo Molecular Technologies, Inc.) and 100 $\mu \mathrm{L}$ medium were added followed by incubation at $37^{\circ} \mathrm{C}$ for $2 \mathrm{~h}$. The OD values were measured using an absorbance microplate reader (Epoch, BioTek, USA) at $450 \mathrm{~nm}$.

\section{LIVE/DEAD Viability Staining}

The cell viability was assessed using a live/dead staining kit (L-7011; Invitrogen, Eugene, OR, USA). After coculture for 3, 7 and 14 days, the composite hydrogel was rinsed with PBS buffer and stained with $500 \mu \mathrm{L}$ live/dead assay each well. Images were observed using a fluorescence microscope (Olympus IX71, inverted microscope, Nagano, Japan). The live and dead cells were stained green or red, respectively.

\section{Cytoskeleton Actin Filament Staining}

BMSCs cultured on the composite hydrogels were examined with FITC-phalloidin assays (Yeasen, Shanghai, China) to observe the morphology and cytoskeleton structure. BMSCs were co-cultured on the composite hydrogels for three days. The DMEM medium was then removed, washed with PBS buffer and fixed in 4\% paraformaldehyde for $10 \mathrm{~min}$. Next, washed with PBS again and immersed in $0.5 \%$ triton $\mathrm{X}-100$ for $5 \mathrm{~min}$. The samples were next treated with $500 \mu \mathrm{L} /$ well of FITC-phalloidin 
$(100 \mathrm{nM}$ ) for $30 \mathrm{~min}$ and with $200 \mu \mathrm{L} /$ well DAPI (Dojindo Laboratories, Kumamoto, Japan) for $30 \mathrm{~s}$. After staining, rinsed with PBS again and visualized with a fluorescence microscope (Olympus IX71, inverted microscope, Nagano, Japan) at $496 \mathrm{~nm}$ (Green, FITC) and $364 \mathrm{~nm}$ (blue, DAPI) wavelengths, respectively.

\section{Alkaline Phosphatase (ALP) Activity}

The composite hydrogels co-cultured with BMSCs for 3 to 14 days. $1 \mathrm{~mL}$ of RIPA and $10 \mu \mathrm{L}$ of PMSF (Solarbio Life Sciences, Beijing, China) were mixed, and $100 \mu \mathrm{L}$ of the mixtures were added to each well for $15 \mathrm{~min}$ on ice to make them completely lysed. The samples were then centrifuged at a speed of $8000 \mathrm{rpm}$ for $20 \mathrm{~min}$ at $4^{\circ} \mathrm{C}$. The supernatant was then evaluated with an ALP assay kit (Beyotime, China). The ALP activity was determined using an absorbance microplate reader. Finally, the ALP levels were normalized against the cellular total protein contents using a bicinchoninic acid (BCA) protein assay kit (Beyotime, China).

\section{ALP and Alizarin Red S Staining}

ALP and Alizarin Red S (ARS, Beijing Solarbio Science \& Technology Co., Ltd.) staining were used to study the mineralization nodules in vitro. BMSCs were seeded in osteoinductive media (Cyagen, China) for 14 days. The samples were fixed with $4 \%$ paraformaldehyde for $30 \mathrm{~min}$ in the dark, then removed and washed with PBS water twice. Finally, ALP staining solution was added and for subsequent optical observation. Similarly, ARS solution was added and incubated for $5 \mathrm{~min}$ at room temperature. Images were obtained with an optical microscope. The stains were dissolved with $5 \%$ perchloric acid for 30 min to quantify the amount of bound ARS, and absorbance was detected at $490 \mathrm{~nm}$.

\section{Quantitative Real-Time PCR}

After co-culture with the composite hydrogels for 3 to 14 days, the total RNA of BMSCs was extracted using NucleoZOL (MACHEREY-NAGEL, Germany). Then the first-strand complementary DNA (cDNA) was synthesized from total RNA using a Hiscript II Q RT SuperMix reagent Kit (Vazyme, China) to obtain the reverse transcription mRNA. Finally, real-time PCR of total $10 \mu \mathrm{L}$ samples in a 96-well PCR plate (Nest Biotechnology, Wuxi, China) was tested using an SYBR Green Master Mix kit (Vazyme, China). To normalize the results, the $2^{-\Delta \Delta \mathrm{Ct}}$ method (Livak's method) was introduced to quantify the gene expression levels. All samples were analyzed in triplicate. Reverse transcription (RT)-PCR primers of osteogenic genes are shown in Table 1.

\section{In vivo Osteogenesis Evaluation of Composite Hydrogel Scaffolds}

A rabbit tibia plateau defect model was applied to study the osteogenic capacity of the composite hydrogel. Animal experiments and care procedures were approved by the Nanjing Medical University Ethics Committee and followed the Guide for the Care and Use of Laboratory Animals (eighth edition).

\section{Composite Hydrogel Scaffolds Implantation}

New Zealand white rabbits (weighing about $2500 \mathrm{~g}$ ) were used to create a tibia plateau defect in this study. The rabbits were first anesthetized with 3\% sodium pentobarbital. Then the skin was sterilized and separated to expose the tibia plateau, and a defect was made with an $(8 \mathrm{~mm}$ diameter and $4 \mathrm{~mm}$ depth) using an electric drill (Bosch, Germany). Immediately after the bone debris was removed, the tibia plateau defect was rinsed with saline solution; the Gel/SA/nano-ATP composite hydrogel scaffolds were implanted into the defect regions, and the wound was sutured. Four types of composite hydrogel scaffolds were placed into different defect sites: control, 5,10 , and $15 \mathrm{w} / \mathrm{v} \%$ of nano-ATP. Surgeries without scaffold implantation were used as a control group. After 4 to 12 weeks of implantation, all the rabbits were sacrificed,

Table I Primer Sequences for Target Genes

\begin{tabular}{|l|l|l|}
\hline Gene & \multicolumn{1}{|c|}{ Forward Primer } & \multicolumn{1}{|c|}{ Reverse Primer } \\
\hline OCN & 5-ACCATCTTTCTGCTCACTCTGCT & 5-CCTTATTGCCCTCCTGCTTG \\
OPN & 5-TACGACCATGAGATTGGCAGTGA & 5-TATAGGATCTGGGTGCAGGCTGTAA \\
Runx-2 & 5-CTGCAAGCAGTATTTACAACAGAGG & 5-GGCTCACGTCGCTCATCTT \\
Osterix & 5-AGGCCTTTGCCAGTGCCTA & 5-GCCAGATGGAAGCTGTGAAGA \\
GAPDH & 5-CACCACCAACTGCTTAGC & 5-TTCACCACCTTCTTGATGTC \\
\hline
\end{tabular}

Abbreviations: OCN, osteocalcin; OPN, osteopontin; OSX, osterix; Runx2, runt-related transcription factor 2; GAPDH, glyceraldehyde-3-phosphate dehydrogenase. 
and the tibia plateaus were harvested for further image evaluation and histological assays.

\section{Histological Analysis}

The rabbits were euthanized at 4,8 , and 12 weeks after implantation. The tibia plateau specimens were collected, fixed with $10 \%$ formalin solution for three days, and then decalcified in 10\% EDTA solution (Aladdin Chemical Inc., Shanghai, China). This changed every three days for four weeks at $37^{\circ} \mathrm{C}$. After decalcification, all the samples were embedded by paraffin, and sliced into 5 - $\mu$ m-thick sections for hematoxylin and eosin (H\&E) staining. Masson trichrome staining was also used for routine histological assessments, and the slices were then observed with a microscope (Olympus, Japan).

\section{Statistical Analysis}

All data were presented as the means \pm standard deviations (SDs) and analyzed by one-way ANOVA. P-values including $p<0.05\left(^{*}\right), p<0.01\left(^{* *}\right)$ and $p<0.001\left(^{* * *}\right)$ were considered to be statistically significant. All quantifications used image software and high-resolution images. Statistical analysis was conducted using Origin 8.0 (Origin Lab Inc.) and GraphPad Prism 5 software (GraphPad Software Inc.).

\section{Results}

\section{Morphology of Gel/SA/Nano-ATP Composite Hydrogel}

The Gel/SA/nano-ATP composite hydrogel scaffolds were fabricated using a 3D bio-printer (Figure 1B). Representative digital photographs show the printed structure with different grid spacing, shapes, and constructed layers in the composite hydrogels. The surface microtopographies of the composite hydrogel scaffolds are shown in Figure 1C. The scaffolds had a regular grid structure. Particularly, the surface of the control group (no nano-ATP) was smoother than that of the other groups. The non-cross-linked groups had more surface pores than the normal cross-linking groups, which indicated that the porosity of the composite hydrogel decreased after ionic and chemical cross-linking, resulting in a significantly increased mechanical strength.

\section{Rheological Properties of Composite Hydrogel Inks}

The gel is a thermo-sensitive bio-ink, and we further studied the rheological behaviors at different temperatures to investigate the effect of the nano-ATP content on their thermal properties. The results showed that the viscous component (loss modulus, G") of the control group was higher than its elastic component (storage modulus, G') at $26^{\circ} \mathrm{C}$. Furthermore, G' and G' increased during the cooling process, and showed a crossover point (Figure 1D). The crossover temperature is defined as the sol-gel transition temperature, which indicates a transition from a solution state to a gel state as the temperature decreases. ${ }^{52}$

In contrast, the hydrogel inks containing nano-ATP showed different rheological behaviors. Upon addition of nano-ATP, G" was always higher than G' even if the temperature changed; there was no crossover point. Although the shear thinning behavior changed, the mechanical properties and printability of the inks improved during the $3 \mathrm{D}$ bioprinting process.

Next, the rheological properties of Gel/SA/nano-ATP inks with different ratios of nano-ATP were also studied. The resulting rheological data are shown in Figure 1E. The results demonstrated that the viscosity of Gel/SA/nano-ATP inks decreased with increasing the shear rate indicating shearthinning behavior. This behavior was no longer obvious as the nano-ATP content increased similar to other results.

\section{Characterization of Composite Hydrogel Scaffolds}

The FTIR spectra of the Gel/SA/nano-ATP composite hydrogel are shown in Figure 2A. The characteristic bands at $3432 \mathrm{~cm}^{-1}$ correspond to free $-\mathrm{OH}$. The absorption peaks at 2949 and $1624 \mathrm{~cm}^{-1}$ were assigned to $\mathrm{C}-\mathrm{H}$ and $\mathrm{C}=\mathrm{O}$ stretching vibration. The FTIR spectra of the four samples showed no significant difference, which indicated that nano-ATP did not affect the cross-linking reaction of Gel/SA hydrogels. Significantly, a characteristic band of gel hydrogel with a peak at $1543 \mathrm{~cm}^{-1}$ (amide II) was absent in the spectrum suggested the involvement of this group in cross-linking reaction. Figure 2B shows XRD patterns of composite hydrogels with different contents of nano-ATP. The characteristic reflections of nano-ATP at $2 \theta$ values of $8.5^{\circ}, 19.6^{\circ}, 27.8^{\circ}$, and $34.3^{\circ}$, which related to (110), (040), (400) and (102) crystalline planes, respectively, were found in the XRD pattern and consistent with previously reported studies. ${ }^{53,54}$

The results of thermal stability of the composite hydrogels are shown in Figure 2C. As illustrated, the addition of nano-ATP to the composite hydrogels led to better thermal stability and less weight loss versus pure Gel/SA hydrogel at $\mathrm{T}<1000^{\circ} \mathrm{C}$. The weight loss decreased as the content of nano-ATP increased, and samples with $10 \mathrm{w} / \mathrm{v} \%$ and $15 \mathrm{w} /$ 

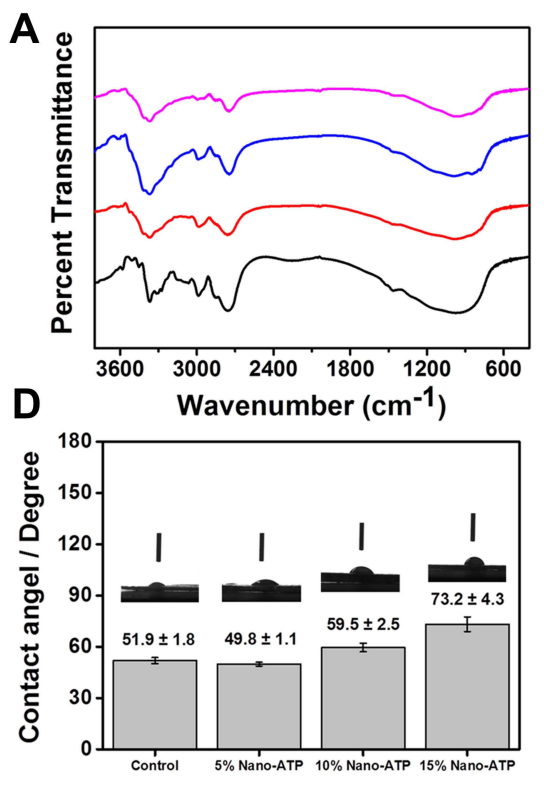

B
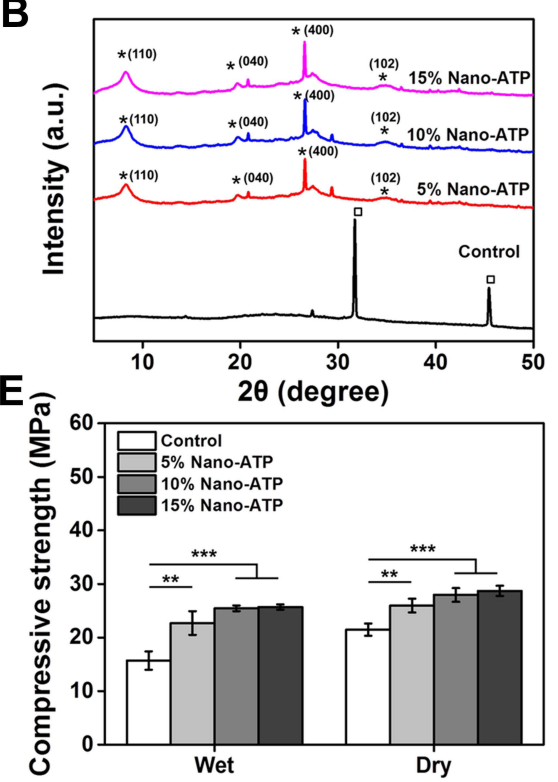

C

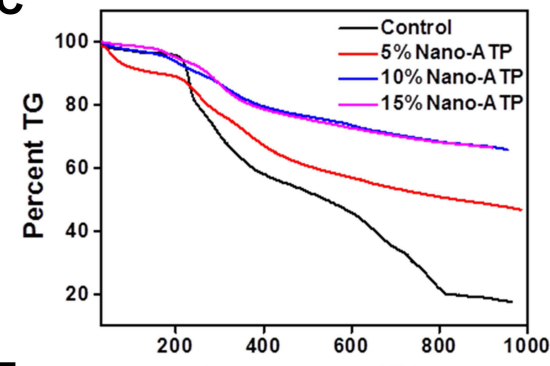

F ชิ

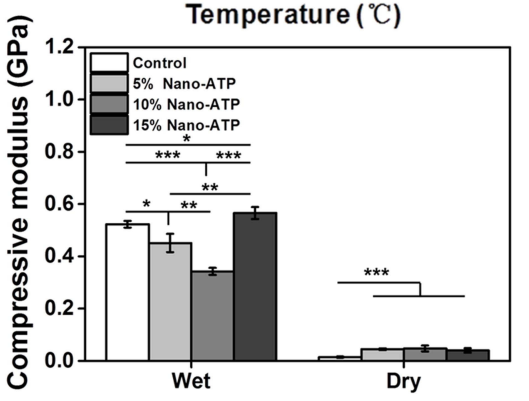

Figure 2 Physicochemical properties of Gel/SA/nano-ATP composite hydrogel scaffolds with different nano-ATP contents. (A) FTIR spectra with characteristic peaks of each compound. (B) XRD patterns of composite hydrogel with different nano-ATP contents with the red dotted line showing the characteristic peaks of nano-ATP. (“*” represents the characteristic peaks of ATP, which related to (II0), (040), (400) and (102) crystalline planes respectively; " $\square$ " represents the characteristic peaks of residual $\mathrm{CaCl}_{2}$ after crosslinking with sodium alginate. (C) TGA curves of the composite hydrogel. Weight loss at $1000{ }^{\circ} \mathrm{C}$ was $82.5 \%, 53.2 \%, 34.1 \%$, and $33.6 \%$, respectively. (D) Photographs of surface contact angle and measurement results of the composite hydrogel. (E) Compressive strength and (F) modulus of Gel/SA/nano-ATP composite hydrogel before (wet) and after being freeze-dried (dry). ( ${ }^{*} p<0.05, * * p<0.01$, ***p $<0.01, \mathrm{n}=5$ ).

Abbreviations: Gel, gelatin; SA, sodium alginate; nano-ATP, nano-attapulgite; FTIR, Fourier transform infrared spectroscopy; XRD, X-ray diffraction; TGA, thermogravimetric analysis.

$\mathrm{v} \%$ content of nano-ATP showed similar thermal stability with weight loss values of $34.2 \%$ and $33.5 \%$, respectively.

The hydrophobicity and hydrophilicity based on the contact angle are essential indicators for materials. The hydrophilicity improves as the water contact angle decreases. The contact angles of the control, 5, 10 and $15 \mathrm{w} / \mathrm{v} \%$ nano-ATP groups were $51.9^{\circ} \pm 1.8^{\circ}, 49.8^{\circ} \pm 1.1^{\circ}, 59.5^{\circ} \pm 2.5^{\circ}$, and $73.2^{\circ}$ $\pm 4.2^{\circ}$, respectively (Figure $2 \mathrm{D}$ ). The $5 \mathrm{w} / \mathrm{v} \%$ nano-ATP samples showed the best hydrophilicity, which decreased with increasing nano-ATP content.

Table 2 shows the zeta potential of both the surface charges of the components of the composite hydrogels and hydrogels in experimental group. The results showed that all single components of the hydrogel and composite hydrogels measured are negatively charged, and the negative charge decreased with the increase of nano-ATP.

\section{Mechanical Properties of Composite Hydrogel Scaffolds}

An ideal bone substitute requires a tissue engineering scaffold with desirable mechanical properties. The compressive strength and modulus of 3D bioprinting Gel/SA/ nano-ATP composite hydrogel with different contents of
nano-ATP were tested in this study. Figure 2E shows the average compressive strengths of the composite hydrogels before (wet) and after being freeze-dried (dry). Gel without nano-ATP (control group) showed the lowest compressive strength of $15.7 \pm 1.7 \mathrm{MPa}$, which was lower than that obtained with $5 \mathrm{w} / \mathrm{v} \%\left(22.7 \pm 2.2 \mathrm{MPa},{ }^{* *} p<0.01\right), 10 \mathrm{w} / \mathrm{v}$ $\%$, and $15 \mathrm{w} / \mathrm{v} \%$ content of nano-ATP $(25.5 \pm 0.1 \mathrm{MPa}$ vs $\left.25.7 \pm 0.5 \mathrm{MPa},{ }^{* * *} p<0.001\right)$ in a wet state. The samples also showed similar results in the dry state after being freeze-dried, this indicated that the compressive strength was increased with nano-ATP content. The dry compressive strength of $5 \mathrm{w} / \mathrm{v} \%$ samples $(25.9 \pm 1.3 \mathrm{MPa})$ were

Table 2 Zeta Potential Analysis (All Data are Given as Mean \pm SD, $\mathrm{n}=3$ )

\begin{tabular}{|l|c|}
\hline Group & Zeta Potential (mv) \\
\hline SA & $-25.17 \pm 4.65$ \\
Gel & $-4.53 \pm 0.7 \mathrm{I}$ \\
Nano-ATP & $-20.20 \pm 0.35$ \\
Control & $-29.23 \pm 1.06$ \\
$5 \%$ Nano-ATP & $-37.97 \pm 0.38$ \\
I0\% Nano-ATP & $-31.37 \pm 0.76$ \\
I5\% Nano-ATP & $-26.93 \pm 1.42$ \\
\hline
\end{tabular}

Abbreviations: Gel, gelatin; SA, sodium alginate; nano-ATP, nano-attapulgite. 
similar to those of $10 \mathrm{w} / \mathrm{v} \%$ and $15 \mathrm{w} / \mathrm{v} \%(27.9 \pm 1.3 \mathrm{MPa}$ vs $28.7 \pm 1.0 \mathrm{MPa}$ ), and all of these were much higher than the control group $(21.5 \pm 1.2 \mathrm{MPa})$.

Figure $2 \mathrm{~F}$ shows the results of the compressive modulus in the dry state. The $15 \mathrm{w} / \mathrm{v} \%$ samples had the highest modulus $(0.566 \pm 0.023 \mathrm{GPa})$, which was higher than the control group $\left(0.523 \pm 0.013 \mathrm{GPa},{ }^{*} p<0.05\right), 5 \mathrm{w} / \mathrm{v} \%(0.451$ $\left.\pm 0.035 \mathrm{GPa},{ }^{* *} p<0.01\right)$, and $10 \mathrm{w} / \mathrm{v} \%(0.342 \pm 0.013 \mathrm{GPa})$ contents of nano-ATP. After being freeze-dried, the modulus decreased significantly due to changes in the properties of the elastomer. The $15 \mathrm{w} / \mathrm{v} \%$ sample showed the highest modulus, which reached about $0.040 \mathrm{GPa}$ and was higher than the control group $(0.015 \pm 0.002 \mathrm{GPa}$, $* * * p<0.001), 5 \mathrm{w} / \mathrm{v} \%(0.045 \pm 0.003 \mathrm{GPa})$, and $10 \mathrm{w} / \mathrm{v} \%$ $(0.047 \pm 0.011 \mathrm{GPa})$ groups.

\section{In vitro Mineralization of Composite Hydrogel Scaffolds in SBF}

For mineralization, the Gel/SA/nano-ATP composite hydrogel was soaked in $1.5 \times$ SBF buffer which was refreshed daily for up to 14 days. Limited mineralization was found on the surface of the control group (Figure 3A) while significant mineralization was found in the composite hydrogels. The mineral deposition was more pronounced when increasing the nano-ATP content.

\section{Swelling of Composite Hydrogel Scaffolds}

Figure 3B shows the influence of nano-ATP concentration on the swelling behavior of the composite hydrogel. A constant increased swelling ratio of the Gel/SA/nanoATP composite hydrogel could be observed until $24 \mathrm{~h}$. The composite hydrogel had a smaller swelling ratio than that of the control group due to the excellent absorption ability of nano-ATP. In addition, as the amount of nano-ATP increased, the swollen scaffolds enable better shape maintenance and mechanical support.

\section{In vitro Degradation Behavior of Composite Hydrogel Scaffolds}

The degradation ability of the bone substitute is also an essential factor in tissue engineering. The degradation behavior of Gel/SA/nano-ATP composite hydrogels was studied in SBF solution at $37^{\circ} \mathrm{C}$. All samples were periodically dried and weighed to calculate their weight loss. Figure $3 \mathrm{C}$ shows the biodegradation results of Gel/SA/nano-ATP composite hydrogels. The control group was degraded by about $40 \%$ after 12 weeks, which is faster than the composite hydrogel with nanoATP. The nano-ATP increased the strength of the hydrogel and made the 3D structure stiffer. Therefore, the Gel/SA/nano-ATP composite hydrogel solved many shortcomings of pure Gel/
A
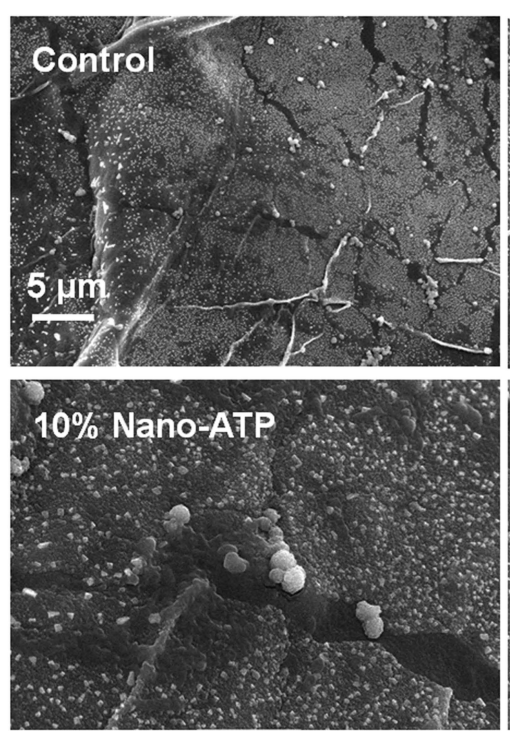
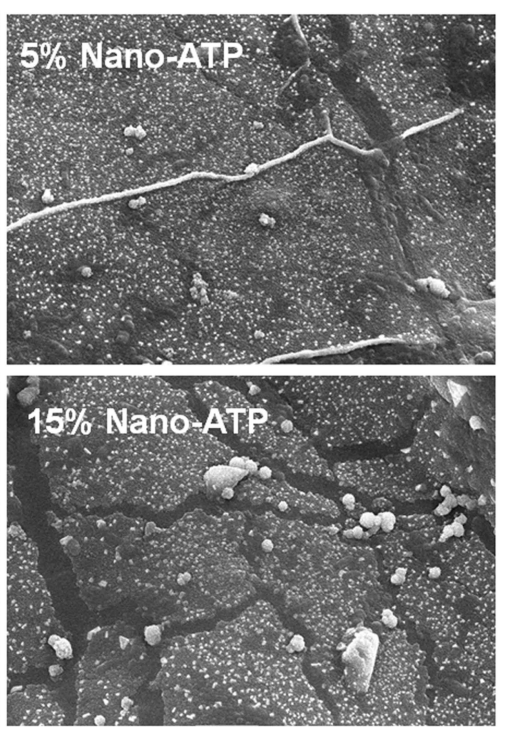

B

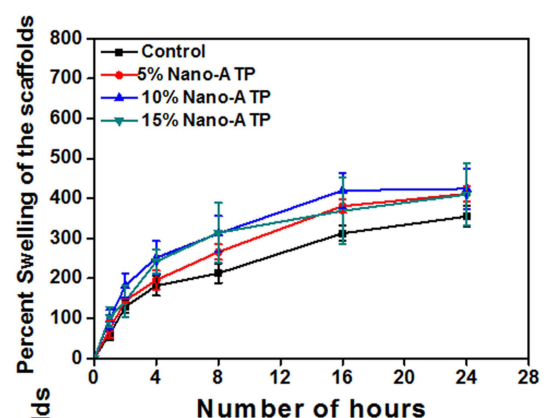

C

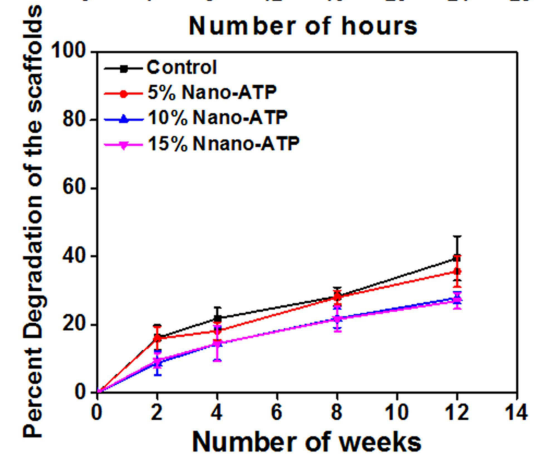

Figure 3 In vitro bio-mineralization and degradation studies. (A) Scanning electron microscopy images of composite hydrogel with different nano-ATP contents obtained after 14 days of incubation in I.5 $\times$ SBF solution with daily replacement of the solution. (B) The swelling ratio of dry composite hydrogels after immersion in deionized water at $37^{\circ} \mathrm{C}$ for $24 \mathrm{~h}$. The error bars represent the standard deviation. (C) In vitro degradation rate of Gel/SA/nano-ATP composite hydrogel with different nano-ATP contents. Error bars represent standard deviation.

Abbreviations: Gel, gelatin; SA, sodium alginate; nano-ATP, nano-attapulgite; SBF, simulated body fluid. 
SA hydrogel; it takes longer time to degrade and provided continuous mechanical support for the defective bone.

\section{Cell Viability of Composite Hydrogel Scaffolds}

The cytotoxicity of the composite hydrogels was measured in vitro. BMSCs cultured in the extract liquor of pure gel and 5, 10, and 15\% nano-ATP of hydrogels for 1,3 and 7 days were compared with that in complete cell culture medium (DMEM). Figure 4A shows the OD values of composite hydrogels with different contents of nano-ATP as well as a culture with DMEM medium as a control group. The results showed that cells in each group proliferated with longer culture times. The DMEM group shows the highest OD values $\left({ }^{*} p<0.05\right)$ on day 7 . The hydrogels contained nano-ATP showed similar OD values versus pure hydrogel. There was no significant difference between them, implying good biocompatibility.

Live/dead staining tested cell viability and cytotoxicity of the 3D bio-printed composite hydrogel. Figure 4B shows that cells seeded on the surface of Gel/SA/nanoATP composite hydrogels attached well after three days of incubation. After seven days of incubation, cells proliferated and covered the entire scaffold surface, hydrogels containing nano-ATP showed better viability than the control group. The $10 \%$ and $15 \%$ nano-ATP hydrogels showed higher viability than 5\% nano-ATP and pure hydrogels and appeared to be the most appropriate formulation for cell culture material.

Cytoskeletal staining was used to study the adhesion and ingrowth of BMSCs after seeding. The cell nucleus was stained with Hoechst (blue), and F-actin was stained with Rhodamine phalloidin (green). After three days of adherence, BMSCs showed increasing numbers and significantly more outstretched filopodia-like extensions versus the control group (Figure 4C).

\section{Osteogenic Differentiation of BMSCs on Composite Hydrogel Scaffolds}

BMSCs in the DMEM, control, and composite hydrogel groups were cultured in their extract medium for 3 to 14 days. ALP staining of the Gel/SA/nano-ATP composite hydrogel groups was deeper than in the control and DMEM groups after 14 days (Figure 5A), and the $10 \%$
A

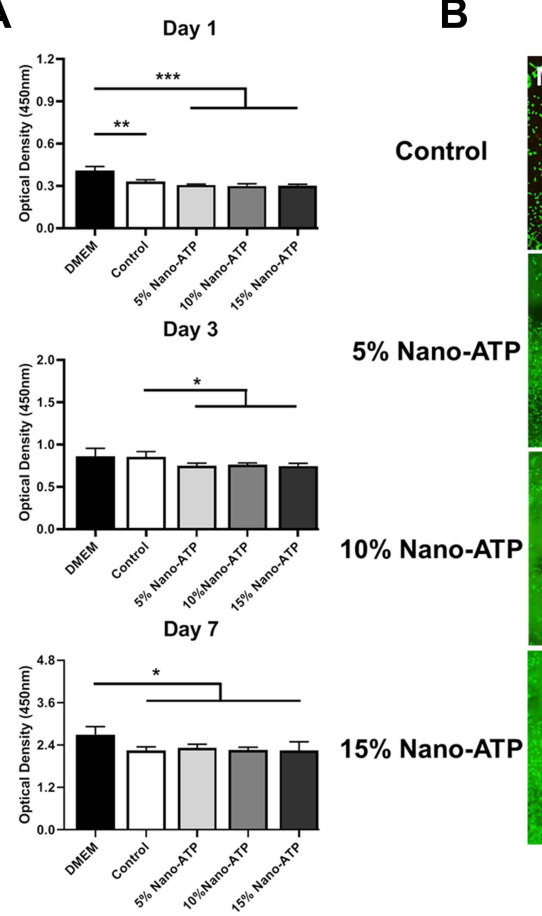

Day 3
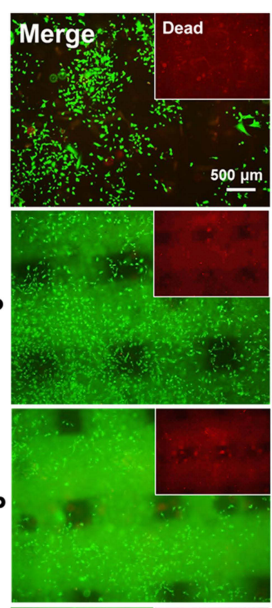

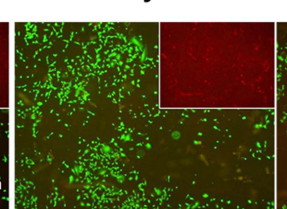

Day 14

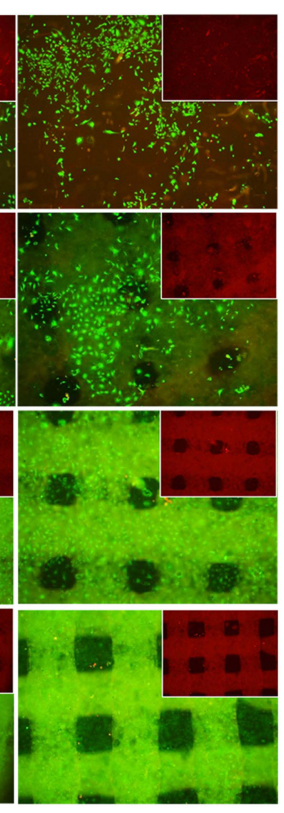

C

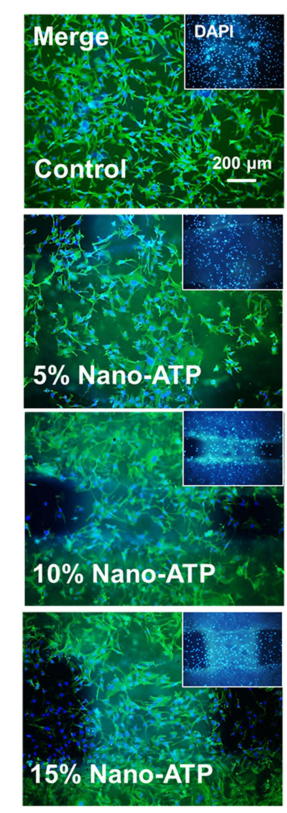

Figure 4 The proliferation and viability of laden cells in vitro. (A) The proliferation of BMSCs cultured within four different composite hydrogel extracts after I, 3, and 7 days using the CCK-8 assay $\left({ }^{*} p<0.05,{ }^{*} p<0.01\right.$, ${ }^{* *} p<0.001, \mathrm{n}=5$ ). (B) Representative merged images of live/dead assays on a cell-laden $3 \mathrm{D}$ bio-printed composite hydrogels at indicated time intervals (3, 7, and 14 days). The living cells are stained green, and dead cells are stained red (scale bar $=500 \mu \mathrm{m}$ ). (C) Fluorescence images obtained after culturing BMSCs for 3 days on composite hydrogels; DAPI was used for the detection of cell nuclei. F-actin was stained green with FITC-phalloidin (Scale bar = $200 \mu \mathrm{m})$.

Abbreviations: nano-ATP, nano-attapulgite; BMSCs, mouse bone marrow-derived mesenchymal stem cells; CCK-8, cell counting kit-8. 

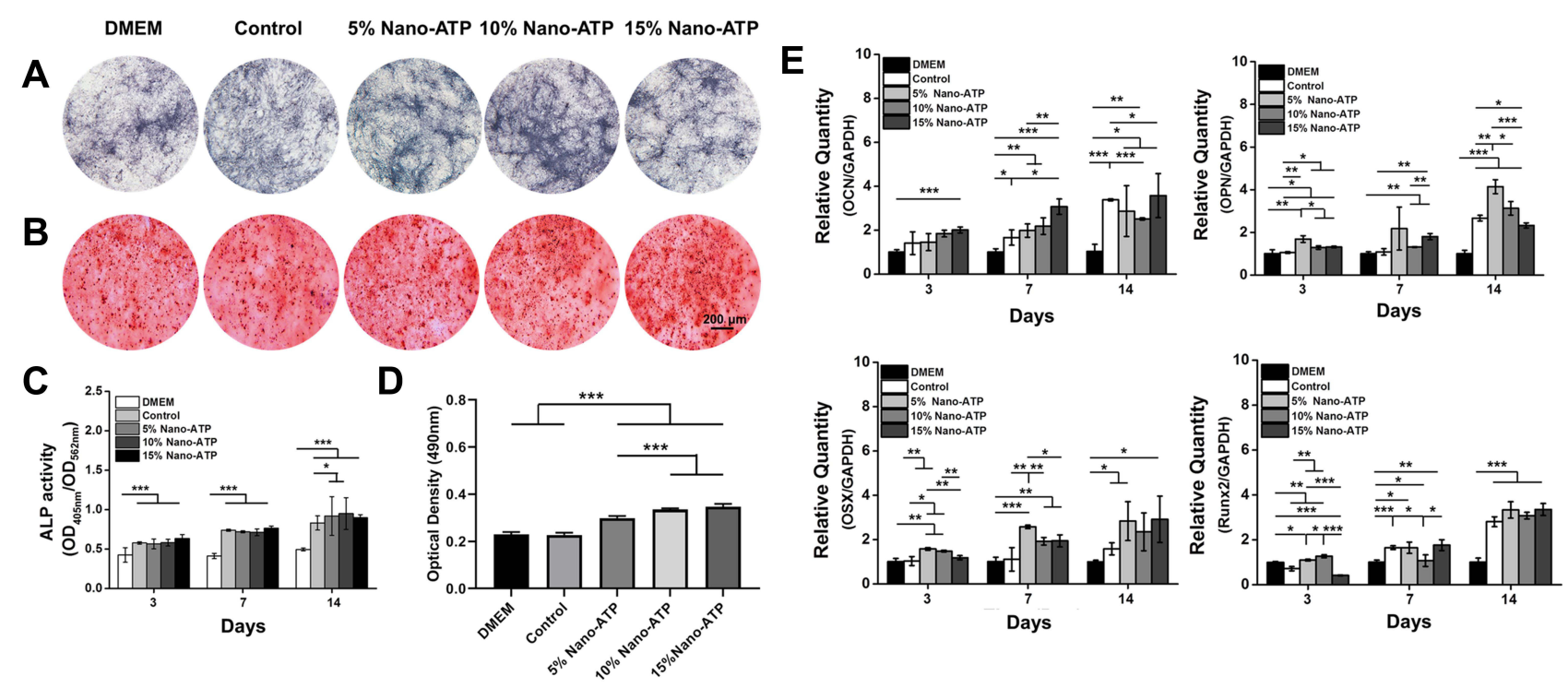

Figure 5 In vitro Gel/SA/nano-ATP composite hydrogel scaffolds promoted osteogenic differentiation. (A) Images of ALP staining and (B) quantification of Alizarin Red $\mathrm{S}$ staining after 14 days of incubation (scale bar $=200 \mu \mathrm{m}$ ). (C) Quantified ALP activity as the ratio between the absorbance at $405 \mathrm{~nm}$ and $562 \mathrm{~nm}$. (D) Quantification of the amount of Alizarin Red S that stained the mineralized matrix. The solution absorbance was detected at $490 \mathrm{~nm}(* * * p<0.00 \mathrm{I}, \mathrm{n}=3)$. (E) Real-time PCR analysis: OCN, OPN, OSX, and Runx2 expressions by BMSCs in the Gel/SA/ATP composite hydrogels after incubation for 3,7 and I4 days. (*p $<0.05, * * p<0.0 \mathrm{I}$, ***p $<0.00 \mathrm{I}, \mathrm{n}=3$ ).

Abbreviations: Gel, gelatin; SA, sodium alginate; nano-ATP, nano-attapulgite; ALP, alkaline phosphatase. PCR, polymerase chain reaction; OCN, osteocalcin; OPN, osteopontin; OSX, osterix; Runx2, runt-related transcription factor 2; GAPDH, glyceraldehyde-3-phosphate dehydrogenase.

group showed the best performance of calcium deposition. ARS staining also showed obvious bonelike nodules and calcium deposition in the composite hydrogel groups versus the control and DMEM groups after co-culture for 14 days (Figure 5B). Figure 5C shows that the composite hydrogels had higher ALP activity than the control group (5\% nano-ATP, ${ }^{*} p<0.05 ; 10 \%$ nano-ATP, ${ }^{*} p<0.05$ ) in 14 days. ARS analysis confirmed that the Gel/SA/nano-ATP composite hydrogel promoted osteogenic differentiation of BMSCs at 14 days versus the control group (Figure 5D), indicating that the Gel/SA/nano-ATP composite hydrogel improved the bioactivity of osteogenesis in the later stage.

Messenger RNA expression levels of $O C N, O P N, O S X$, and Runx 2 were significantly increased for BMSCs cultured on the Gel/SA/nano-ATP composite hydrogel versus the control group. This increase was detected by quantitative real-time PCR assay (Figure 5E). Specifically, the $O C N$, $O P N, O S X$, and Runx2 expressions were all markedly upregulated for cells cultured on the gels with an increase of coculture days. Furthermore, the $O C N$ expression in the case of $15 \%$ nano-ATP was higher than the control group on the 7 th and 14th days $\left({ }^{*} p<0.05\right)$. The $O P N, O S X$, and Runx 2 makers all showed the highest expression values in the $5 \%$ nano-ATP groups than in the other groups on the $3 \mathrm{rd}$, 7th, and 14 th day. However, the Runx2 expression was slightly lower than that in the $15 \%$ nano-ATP group on the 7th day. In addition, the OCN, OSX, and Runx 2 values in the case of $15 \%$ nano-ATP were similar to those with 5\% nano-ATP after co-culture for 14 days, but the difference was not significant.

\section{In vivo Osteogenesis Evaluation of Composite Hydrogel Scaffolds}

Figure 6A shows the tibia bone defects in rabbits implanted with hydrogels before and 4 to 12 weeks after surgery. The sham group (no material) was used as a defect control group. All of the groups were sacrificed, and tibia plateaus were isolated for observation, fixed with $10 \%$ formalin, and decalcified with $10 \%$ EDTA solution for histological evaluation. As shown in Figure 6B and C, $\mathrm{H} \& \mathrm{E}$, and Masson's trichrome staining evaluated the newly formed tissues of bone defect sites treated with Gel/SA/nano-ATP composite hydrogels.

At the 4th week, the space between the defect and Gel/SA/nano-ATP composite hydrogels was filled with matrix, osteoblasts, and immunocytes. The osteoblasts and bone matrix collagen, which stained red and grew into the $15 \%$ nano-ATP hydrogels could be obviously observed in Figure 6B. In contrast, the defects were mostly filled with immunocytes and fibroblasts in the Gel/SA hydrogel and defect control groups. After 8 weeks, new bone formation was found in the Gel/SA/ 


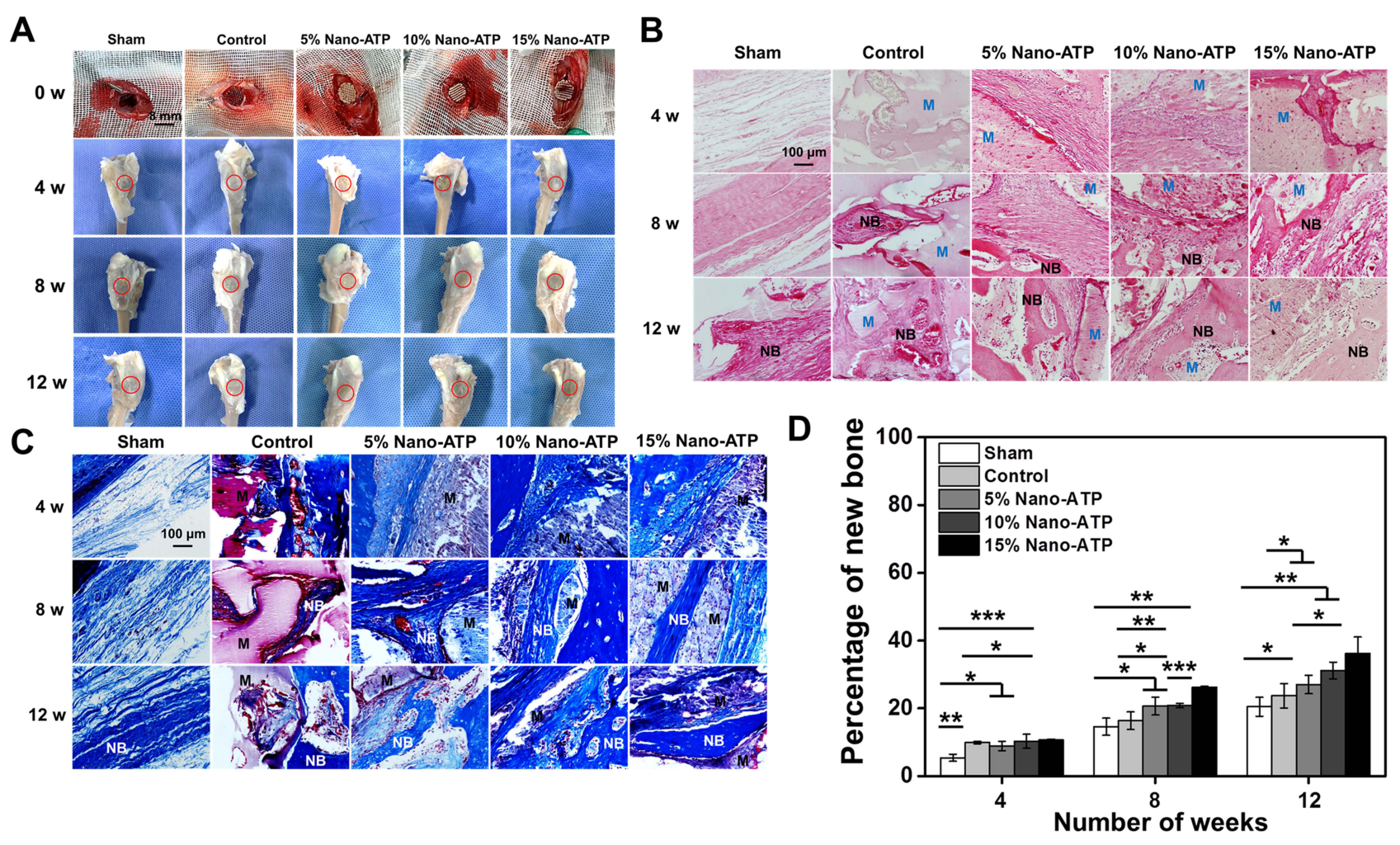

Figure 6 Histological analysis of Gel/SA/nano-ATP composite hydrogel scaffolds in rabbit tibial plateau defects at 4 to 12 weeks post-implantation. (A) Digital photographs of the five experimental groups during surgery (0 weeks) and at 4-12 weeks post-surgery. (B) Staining with H\&E and (C) Masson's trichrome demonstrates new bone formed in the composite hydrogel (scale bar $=100 \mu \mathrm{m})$. (D) Quantitative data of Masson's trichrome staining. $\left({ }^{*} p<0.05, * * p<0.0 \mathrm{I}, * * * p<0.00 \mathrm{I}, \mathrm{n}=3\right.$ ).

Abbreviations: Gel, gelatin; SA, sodium alginate; nano-ATP, nano-attapulgite; H\&E, hematoxylin and eosin; M, implanted material; NB, new bone.

nano-ATP composite hydrogel. On the contrary, only new bone matrix and osteoblasts were seen in the control group with extensive fibrous tissue in the defect control group. More new bone tissue was observed in the space between the defect and materials at 12 weeks after implantation.

Masson's trichrome staining demonstrated new bone formed in the composite hydrogel (Figure 6C). The results showed that the fibrous tissue (blue) filled the defect areas, and there was no observable bone formation in any group on the 4th week. After 8 weeks, osteoblasts and mature bone tissues were clearly seen in the Gel/SA/nano-ATP composite hydrogel. In contrast, only a little new bone grew in the control group, a large amount of fibrous tissue filled in the defect control group. Twelve weeks later, a small amount of new bone grew in the defect control group.

Meanwhile, abundant new bone formation was observed in the Gel/SA/nano-ATP composite hydrogel groups. The scaffold degraded along with the growth of new bone. Quantitative data confirmed that higher nano-
ATP concentrations led to more new bone tissue (Figure 6D).

\section{Discussion}

In this study, we successfully fabricated Gel/SA/nanoATP composite hydrogels loaded with different contents of nano-ATP using 3D printing technology for bone tissue engineering. The nano-ATP is a hydrated layerchain magnesium silicate mineral with unique physicochemical properties. ${ }^{40}$ Several nano-ATP composite materials have been constructed in our previous study, and the results showed that it owns good biocompatibility and potential to promote osteoblastic specification. ${ }^{48,49}$ After adding nano-ATP, the G" (loss modulus) of the Gel/SA/ nano-ATP inks was always lower than the $G^{\prime}$ (storage modulus) independent of temperature changes. The crossover point further disappeared. The effect of temperature on the ink was weakened and changed the shear thinning behavior, but the state of inks was controllable, and the printability of the composite hydrogel inks during the 3D bioprinting process was demonstrated (Figure 1D). Figure 1E shows that the viscosity of inks increased as 
the nano-ATP content increased. The shear-thinning behavior was no longer obvious, which corresponds to previous results. ${ }^{48,49}$

Although the contact angles of the composite hydrogel were close to or over 50 degrees, it would not have a great effect on cell attachment, proliferation, and differentiation. That is because combining alginate and gelatin facilitates cell adherence to the surface of the scaffold, and the nanoATP could act directly as focal adhesion sites through the provision of reactive functional groups for cell attachment due to its special rod crystal structure. ${ }^{55}$ Additionally, abundant $\mathrm{Si}$ and $\mathrm{Mg}$ in nano-ATP could stimulate the osteogenic differentiation of bone stem cells. The results in this paper were consistent with the previous research results, which proved that nano-ATP has an effect on promoting cell proliferation and differentiation. ${ }^{56}$ The mechanical properties of the composite hydrogels play a significant role in BTE. ${ }^{57,58}$ The mechanical properties were remarkably improved after adding nano-ATP to the Gel/SA hydrogel while the degradation rate decreased. The compressive strength of $15 \mathrm{w} / \mathrm{v} \%$ nano-ATP was higher than that of Gel/SA hydrogel and increased by about $10 \mathrm{MPa}$ (Figure 2E). The Gel/SA/nano-ATP hydrogel degraded more slowly than the control group, and the magnitude of degradation depends on the content of nanoATP. At 12 weeks, the degradation rate of the control group was twice that of the $15 \mathrm{w} / \mathrm{v} \%$ nano-ATP group in vitro. The ideal situation in bone defect repair is that the new bone forms with the degradation of the scaffold. ${ }^{59}$ Therefore, the Gel/SA/nano-ATP hydrogel scaffold has the potential for repairing bone defects and could provide sufficient mechanical support for the defect site with controllable biodegradability.

A series of characterization results showed that the physical-chemical properties of the Gel/SA hydrogel changed after the addition of nano-ATP (Figure 2A and B). The weight loss decreased as the nano-ATP content increased; the thermal stability in the high-temperature region was improved. Similarly, the hydrophilicity and swelling of the hydrogel increased because nano-ATP has good water absorption properties. Similar to previous studies, the rapid swelling behavior of the scaffolds promoted cell adsorption and growth. ${ }^{60}$ Good bioactivity is also an important factor for bone tissue engineering scaffolds. ${ }^{58}$ After 14 days of incubation in $1.5 \times \mathrm{SBF}$ solution, an apatite-like layer was observed in the SEM images (Figure 3A). In addition, the amount of hydroxyapatite increased on the surface of the hydrogels with increasing
nano-ATP concentration. The results showed that the Gel/ SA/nano-ATP composite hydrogels had excellent bioactivity.

As a basic requirement for BTE, the biocompatibility of a scaffold is often the primary factor to evaluate whether it could provide a suitable microenvironment for cell survival and proliferation. ${ }^{61}$ Hydrogels containing nano-ATP showed good biocompatibility. The gel and alginate had good biocompatibility, and the nano-ATP was nontoxic with good biocompatibility for a bone tissue scaffold. The results of live/dead and cytoskeletal staining (Figure 3B and C) further demonstrated that the composite hydrogel not only provided a proper microenvironment for BMSC survival but also promoted proliferation and stretching of BMSCs versus the control hydrogel. Furthermore, 3D printing technology could be used to fabricate appropriate pore sizes $(\sim 400 \mu \mathrm{m})$ and porosities of the composite hydrogels for bone defect repair, which match the recommended size of 100-400 $\mu \mathrm{m}$ for osteoconduction. $^{62,63}$ They also provide enough surface area, pore interconnections, and channels for cell growth and nutrient transport. The results indicated that the addition of nano-ATP did not affect cell viability, and the Gel/SA/ nano-ATP composite hydrogel met the requirements of bone defect repair.

The Gel/SA/nano-ATP composite hydrogel could promote osteogenic differentiation of BMSCs in vitro, which is consistent with previous studies. ${ }^{64}$ The ALP activity of BMSCs increased after 14 days, suggesting that the Gel/SA/ nano-ATP composite hydrogel can induce an osteogenic response in BMSCs. Furthermore, ARS staining showed obvious extracellular calcium deposits produced by osteoblasts in the Gel/SA/nano-ATP composite hydrogels versus the gel without nano-ATP. These results indicated that the addition of nano-ATP could efficiently induce the differentiation and mineralization of BMSCs. The relative expression of the osteogenic differentiation-related genes $O C N, O P N$, OSX, and Runx 2 was significantly higher in the $5 \mathrm{w} / \mathrm{v} \%$ nanoATP groups than in the control group on the 3rd, 7th, and 14th day. Hence, the increase in osteoblastic marker expression further confirmed the differentiation of BMSCs grown on the Gel/SA/nano-ATP composite hydrogel scaffold.

The specific mechanism by which nano-ATP promotes osteogenic differentiation of osteoblast precursor cells remains unclear, but the osteogenic effects seem to be mainly attributed to the abundant $\mathrm{Si}$ and $\mathrm{Mg}$ in nanoATP. Thevenot et al mentioned that the incorporation of negative charges may facilitate adsorption of proteins that promote cell adhesion and responses, ${ }^{65,66}$ so the addition 
of negatively charged ATP may have the same effects. Some studies have shown that $\mathrm{Si}$ and $\mathrm{Mg}$ ions could stimulate the osteogenic differentiation of bone stem cells and can also promote vascularization. ${ }^{55,67}$ In addition, nano-ATP has special rod-like crystal structures that can act as focal adhesion sites and provide reactive functional groups to facilitate cell attachment and spreading. ${ }^{68}$ Another possible mechanism may be that $\mathrm{Si}^{4+}$ or $\mathrm{Mg}^{2+}$ preferentially exchange on ATP particle surfaces relative to monovalent ions when the local concentrations of divalent cations increase with the charge density. These relevant ions are necessary for the function of integrins, and the transmembrane receptors could mediate cell interactions with ECM. ${ }^{69}$ However, more research is needed to explore the detailed mechanism of nano-ATP on BMSCs.

The results of the tibia bone defect showed that more new bone tissue grew in defects with a higher nano-ATP concentration in the hydrogels. At the 8th and 12 th weeks (Figure 6B and $\mathrm{C}$ ), there were new bone tissues within the pores in the Gel/SA/nano-ATP composite hydrogels, which was consistent with the mechanism proposed above. The results in this study illustrate that the Gel/SA/ nano-ATP composite hydrogel has good compatibility and degradability and could promote bone repair and regeneration, thus demonstrating a promising potential bone tissue scaffold for bone repair.

\section{Conclusion}

In this study, Gel/SA/nano-ATP composite hydrogels containing different nano-ATP contents for bone repair were fabricated using 3D bioprinting technology. The Gel/SA/ nano-ATP composite hydrogel showed better printability and mechanical properties compared to the gel without nano-ATP. In addition, the composite hydrogel showed good biocompatibility and could promote osteogenesis differentiation of BMSCs. Furthermore, there were obvious new bone tissues within the pores in the Gel/SA/nano-ATP composite hydrogel scaffolds after implantation in a rabbit tibia bone defect for 12 weeks; this indicates that they were more effective in inducing bone regeneration than the control group. To summarize, the Gel/SA/nano-ATP composite hydrogel scaffolds could repair bone defects.

\section{Acknowledgments}

This study was supported by the Key Project of Science and Technology of Jiangsu Province (Grant BE2018644), Science and Technology Support Plan of Changzhou City (Social Development) (Grant CE20185047), Young
Talents Science and Technology project of Changzhou Health Commission (Grant QN202029), and the Changzhou Sci\&Tech Program (Grant CJ20210102).

\section{Disclosure}

The authors declare that there is no conflict of interest regarding the publication of this paper.

\section{References}

1. Wang J, Yin Q, Gu S, Wu Y, Rui Y. Induced membrane technique in the treatment of infectious bone defect: a clinical analysis. Orthop Traumatol Surg Res. 2019;105(3):535-539.

2. Zhang T, Wei Q, Zhou H, et al. Three-dimensional-printed individualized porous implants: a new "implant-bone" interface fusion concept for large bone defect treatment. Bioact Mater. 2021;6 (11):3659-3670. doi:10.1016/j.bioactmat.2021.03.030

3. Wang X, Luo F, Huang K, Xie Z. Induced membrane technique for the treatment of bone defects due to post-traumatic osteomyelitis. Bone Joint Res. 2016;5(3):101-105. doi:10.1302/2046-3758.53. 2000487

4. Schmidt AH. Autologous bone graft: is it still the gold standard? Injury. 2021;52:S18-S22. doi:10.1016/j.injury.2021.01.043

5. Scalzone A, Flores-Mir C, Carozza D, d'Apuzzo F, Grassia V, Perillo L. Secondary alveolar bone grafting using autologous versus alloplastic material in the treatment of cleft lip and palate patients: systematic review and meta-analysis. Prog Orthod. 2019;20(1):1-10. doi:10.1186/s40510-018-0252-y

6. Sharmin F, O'Sullivan M, Malinowski S, Lieberman JR, Khan Y. Large scale segmental bone defect healing through the combined delivery of VEGF and BMP-2 from biofunctionalized cortical allografts. J Biomed Mater Res B Appl Biomater. 2019;107 (4):1002-1010. doi:10.1002/jbm.b.34193

7. Utomo DN, Hernugrahanto KD, Edward M, Widhiyanto L, Mahyudin F. Combination of bone marrow aspirate, cancellous bone allograft, and platelet-rich plasma as an alternative solution to critical-sized diaphyseal bone defect: a case series. Int J Surg Case Rep. 2019;58:178-185. doi:10.1016/j.ijscr.2019.04.028

8. Qu H, Fu H, Han Z, Sun Y. Biomaterials for bone tissue engineering scaffolds: a review. $R S C A d v$. 2019;9(45):26252-26262. doi:10.1039/ C9RA05214C

9. Koons GL, Diba M, Mikos AG. Materials design for bone-tissue engineering. Nat Rev Mater. 2020;5(8):584-603.

10. Xu C, Bai Y, Yang S, et al. A versatile three-dimensional foam fabrication strategy for soft and hard tissue engineering. Biomed Mater. 2018;13(2):025018. doi:10.1088/1748-605X/aaalf6

11. Xu C, Liu H, Yang H, Yang L. A green biocompatible fabrication of highly porous functional ceramics with high strength and controllable pore structures. $J$ Mater Sci Technol. 2016;32(8):729-732. doi:10.1016/j.jmst.2016.07.002

12. Liu Y, Xu C, Gu Y, et al. Polydopamine-modified poly (1-lactic acid) nanofiber scaffolds immobilized with an osteogenic growth peptide for bone tissue regeneration. RSC $A d v$. 2019;9(21):11722-11736. doi:10.1039/C8RA08828D

13. Xiao Y, Yang Y, Li J, et al. Porous composite calcium citrate/polylactic acid materials with high mineralization activity and biodegradability for bone repair tissue engineering. Int J Polym Mater Polym Biom. 2021;70(7):507-520. doi:10.1080/00914037.2020.1740984

14. Haugen HJ, Lyngstadaas SP, Rossi F, Perale G. Bone grafts: which is the ideal biomaterial? J Clin Periodontol. 2019;46:92-102. doi:10.1111/jcpe. 13058

15. Liu W. 3D Printing of Multi-Functional Hydrogels. Johns Hopkins University; 2019. 
16. Sun $\mathrm{H}, \mathrm{Hu} \mathrm{C}$, Zhou $\mathrm{C}$, et al. 3D printing of calcium phosphate scaffolds with controlled release of antibacterial functions for jaw bone repair. Mater Des. 2020;189:108540. doi:10.1016/j.matdes.2020.108540

17. Haleem A, Javaid M, Khan RH, Suman R. 3D printing applications in bone tissue engineering. J Clin Orthop Trauma. 2020;11:S118S124. doi:10.1016/j.jcot.2019.12.002

18. Xu C, Thiruvadi VS, Whitmore R, Liu H. Delivery systems for biomedical applications: basic introduction, research frontiers and clinical translations. In: Biomaterials in Translational Medicine. Elsevier; 2019:93-116.

19. Rahighi R, Panahi M, Akhavan O, Mansoorianfar M. Pressure-engineered electrophoretic deposition for gentamicin loading within osteoblast-specific cellulose nanofiber scaffolds. Mater Chem Phys. 2021;272:125018. doi:10.1016/j.matchemphys.2021.125018

20. Li J, Wu C, Chu PK, Gelinsky M. 3D printing of hydrogels: rational design strategies and emerging biomedical applications. Mater Sci Eng R Rep. 2020;140:100543.

21. Distler T, Boccaccini AR. 3D printing of electrically conductive hydrogels for tissue engineering and biosensors - a review. Acta biomaterialia. 2020;101:1-13. doi:10.1016/j.actbio.2019.08.044

22. Xu C, Hung C, Cao Y, Liu HH. Tunable crosslinking, reversible phase transition, and 3D printing of hyaluronic acid hydrogels via dynamic coordination of innate carboxyl groups and metallic ions. ACS Appl Bio Mater. 2021;4(3):2408-2428. doi:10.1021/acsabm.0c01300

23. $\mathrm{Xu} \mathrm{C}$, Wei $\mathrm{Z}$, Gao $\mathrm{H}$, et al. Bioinspired mechano-sensitive macroporous ceramic sponge for logical drug and cell delivery. Adv Sci. 2017;4(6):1600410. doi:10.1002/advs.201600410

24. Xu C, Bai Y, Yang H, Yang L. Mechanically modulated, ultra-high precision logic delivery of molecules by bio-inspired macroporous ceramic sponge. $M R S A d v$. 2017;2(19-20):1125-1130. doi:10.1557/adv.2017.87

25. Akhavan O, Ghaderi E, Shirazian SA, Rahighi RJC. Rolled graphene oxide foams as three-dimensional scaffolds for growth of neural fibers using electrical stimulation of stem cells. Carbon. 2016;97:71-77.

26. Yoon H, Ahn S, Kim G. Three-dimensional polycaprolactone hierarchical scaffolds supplemented with natural biomaterials to enhance mesenchymal stem cell proliferation. Macromol Rapid Commun. 2009;30(19):1632-1637. doi:10.1002/marc.200900264

27. Sun Y, Xing Z, Xue Y, Mustafa K, Finne-Wistrand A, Albertsson AC. Surfactant as a critical factor when tuning the hydrophilicity in three-dimensional polyester-based scaffolds: impact of hydrophilicity on their mechanical properties and the cellular response of human osteoblast-like cells. Biomacromolecules. 2014;15(4):1259-1268. doi:10.1021/bm401818e

28. de Lima EL, Vasconcelos NF, da Silva Maciel J, Andrade FK, Vieira RS, Feitosa JPA. Injectable hydrogel based on dialdehyde galactomannan and $\mathrm{N}$-succinyl chitosan: a suitable platform for cell culture. J Mater Sci Mater Med. 2020;31(1):1-13.

29. Gao J, Ding X, Yu X, et al. Cell-free bilayered porous scaffolds for osteochondral regeneration fabricated by continuous 3D-printing using nascent physical hydrogel as ink. Adv Healthcare Mater. 2021;10(3):2001404. doi:10.1002/adhm.202001404

30. Chan K, Zhuo S, Ni M. Natural and synthetic peptide-based biomaterials for bone tissue engineering. OA Tiss Eng. 2013;1(1):6.

31. Chan KH, Lee WH, Zhuo S, Ni MJ. Harnessing supramolecular peptide nanotechnology in biomedical applications. Int J Nanomed. 2017;12:1171. doi:10.2147/IJN.S126154

32. Freeman FE, Kelly DJ. Tuning alginate bioink stiffness and composition for controlled growth factor delivery and to spatially direct MSC fate within bioprinted tissues. Sci Rep. 2017;7(1):1-12. doi:10.1038/ s41598-017-17286-1

33. Zhou L, Fan L, Zhang F-M, et al. Hybrid gelatin/oxidized chondroitin sulfate hydrogels incorporating bioactive glass nanoparticles with enhanced mechanical properties, mineralization, and osteogenic differentiation. Bioact Mater. 2021;6(3):890-904. doi:10.1016/j. bioactmat.2020.09.012
34. Gupta D, Kocot M, Tryba AM, et al. Novel naturally derived whey protein isolate and aragonite biocomposite hydrogels have potential for bone regeneration. Mater Des. 2020;188:108408. doi:10.1016/j. matdes.2019.108408

35. Watanabe M, Li H, Yamamoto M, Horinaka J, Tabata Y, Flake AW. Addition of glycerol enhances the flexibility of gelatin hydrogel sheets; application for in utero tissue engineering. J Biomed Mater Res B Appl Biomater. 2021;109(6):921-931. doi:10.1002/jbm.b.34756

36. Xu J, Wang Z, You J, et al. Polymerization of moldable self-healing hydrogel with liquid metal nanodroplets for flexible strain-sensing devices. Chem Eng J. 2020;392:123788. doi:10.1016/j.cej.2019. 123788

37. Jang T-S, Jung H-D, Pan HM, Han WT, Chen S, Song J. 3D printing of hydrogel composite systems: recent advances in technology for tissue engineering. Int $J$ Bioprinting. 2018;4(1). doi:10.18063/ijb. v4i1.126

38. Gao T, Gillispie GJ, Copus JS, et al. Optimization of gelatin-alginate composite bioink printability using rheological parameters: a systematic approach. Biofabrication. 2018;10(3):034106. doi:10.10 88/1758-5090/aacdc7

39. Di Giuseppe M, Law N, Webb B, et al. Mechanical behaviour of alginate-gelatin hydrogels for 3D bioprinting. J Mech Behav Biomed Mater. 2018;79:150-157. doi:10.1016/j.jmbbm.2017.12.018

40. Wang W, Wang A. Recent progress in dispersion of palygorskite crystal bundles for nanocomposites. Appl Clay Sci. 2016;119:18-30. doi:10.1016/j.clay.2015.06.030

41. Akhavan O, Ghaderi E, Shahsavar MJC. Graphene nanogrids for selective and fast osteogenic differentiation of human mesenchymal stem cells. Carbon. 2013;59:200-211.

42. Dawson JI, Oreffo RO. Clay: new opportunities for tissue regeneration and biomaterial design. Adv Mater. 2013;25(30):4069-4086. doi:10.1002/adma.201301034

43. Wang W, Wang F, Kang Y, Wang A. Nanoscale dispersion crystal bundles of palygorskite by associated modification with phytic acid and high-pressure homogenization for enhanced colloidal properties. Powder Technol. 2015;269:85-92. doi:10.1016/j. powtec.2014.09.015

44. Duan Z, Zhao Q, Wang S, et al. Novel application of attapulgite on high performance and low-cost humidity sensors. Sens Actuators B Chem. 2020;305:127534. doi:10.1016/j.snb.2019.127534

45. Dong L, Wang H, Huang Y, et al. Elemental mercury removal from coal-fired flue gas using recyclable magnetic $\mathrm{Mn}-\mathrm{Fe}$ based attapulgite sorbent. Chem Eng J. 2021;407:127182. doi:10.1016/j.cej.2020. 127182

46. Wang Y, Zhou R, Wang C, et al. Novel environmental-friendly nano-composite magnetic attapulgite functionalized by chitosan and EDTA for cadmium (II) removal. J Alloys Compd. 2020;817:153286. doi:10.1016/j.jallcom.2019.153286

47. Dong HY, Buard A, Renier A, Lévy F, Saint-Etienne L, Jaurand M-C. Role of oxygen derivatives in the cytotoxicity and DNA damage produced by asbestos on rat pleural mesothelial cells in vitro. Carcinogenesis. 1994;15 (6):1251-1255. doi:10.1093/carcin/15.6.1251

48. Ning Y, Qin W, Ren Y, Li C, Chen W, Zhao H. Effect of icariin/ attapulgite/collagen type I/polycaprolactone composite scaffold in repair of rabbit tibia defect. Zhongguo Xiu Fu Chong Jian Wai Ke Za Zhi= Zhongguo Xiufu Chongjian Waike Zazhi= Chinese Journal of Reparative and Reconstructive Surgery. 2019;33(9):1181-1189. doi:10.7507/1002-1892.201902044

49. Zhang XM, Wang SY, Li G, Zhao HB. The study of osteogenic induction of type I collagen/poly (caprolactone)/attapulgite composite scaffold materials in vitro. China Biotechnol. 2016;36(5):27-33.

50. Sujan MI, Sarkar SD, Sultana S, et al. Bi-functional silica nanoparticles for simultaneous enhancement of mechanical strength and swelling capacity of hydrogels. RSC Adv. 2020;10(11):6213-6222. doi:10.1039/C9RA09528D 
51. Jiang LB, Su DH, Ding SL, et al. Salt-assisted toughening of protein hydrogel with controlled degradation for bone regeneration. $A d v$ Funct Mater. 2019;29(26):1901314. doi:10.1002/adfm.201901314

52. Bielejewski M, Łapiński A, Demchuk O. Molecular interactions in high conductive gel electrolytes based on low molecular weight gelator. $J$ Colloid Interface Sci. 2017;490:279-286. doi:10.1016/j. jcis.2016.11.059

53. Wang Z, Hui A, Zhao H, et al. A novel 3D-bioprinted porous nano attapulgite scaffolds with good performance for bone regeneration. Int JNanomed. 2020;15:6945. doi:10.2147/IJN.S254094

54. Li W, Liu Y, Zhang Q, Tan Z, Jia S Effect of CaCl2 on the stability and antimicrobial activity of nisin. Paper presented at: IOP Conference Series: Earth and Environmental Science; 2021.

55. Mousa M, Evans ND, Oreffo RO, Dawson JI. Clay nanoparticles for regenerative medicine and biomaterial design: a review of clay bioactivity. Biomaterials. 2018;159:204-214. doi:10.1016/j. biomaterials.2017.12.024

56. Zhao H, Zhang X, Zhou D, et al. Collagen, polycaprolactone and attapulgite composite scaffolds for in vivo bone repair in rabbit models. Biomed Mat. 2020;15(4):045022. doi:10.1088/1748-605X/ab843f

57. Mondal S, Nguyen TP, Hoang G, et al. Hydroxyapatite nano bioceramics optimized 3D printed poly lactic acid scaffold for bone tissue engineering application. Ceram Int. 2020;46(3):3443-3455. doi:10.1016/j.ceramint.2019.10.057

58. Chuan D, Fan R, Wang Y, et al. Stereocomplex poly (lactic acid)-based composite nanofiber membranes with highly dispersed hydroxyapatite for potential bone tissue engineering. Compos Sci Technol. 2020;192:108107. doi:10.1016/j.compscitech.2020.108107

59. Park S-H, Gil ES, Shi H, Kim HJ, Lee K, Kaplan DL. Relationships between degradability of silk scaffolds and osteogenesis. Biomaterials. 2010;31(24):6162-6172. doi:10.1016/j.biomaterials.20 10.04 .028

60. Matyash M, Despang F, Ikonomidou C, Gelinsky M. Swelling and mechanical properties of alginate hydrogels with respect to promotion of neural growth. Tissue Eng Part C Methods. 2014;20 (5):401-411. doi:10.1089/ten.tec.2013.0252
61. Xue X, Hu Y, Deng Y, Su J. Recent advances in design of functional biocompatible hydrogels for bone tissue engineering. Adv Funct Mater. 2021;31(19):2009432. doi:10.1002/adfm.202009432

62. Xie X, Shi X, Wang S, Cao L, Yang C, Ma Z. Effect of attapulgite-doped electrospun fibrous PLGA scaffold on pro-osteogenesis and barrier function in the application of guided bone regeneration. Int J Nanomedicine. 2020;15:6761. doi:10.2147/ IJN.S244533

63. Jones AA, Buser D, Schenk R, Wozney J, Cochran DL. The effect of rhBMP-2 around endosseous implants with and without membranes in the canine model. $J$ Periodontol. 2006;77(7):1184-1193. doi:10.1902/jop.2006.050337

64. Hing KA, Wilson LF, Buckland T. Comparative performance of three ceramic bone graft substitutes. Spine J. 2007;7(4):475-490. doi:10.1016/j.spinee.2006.07.017

65. Chang HI, Wang Y. Cell responses to surface and architecture of tissue engineering scaffolds. In: Regenerative Medicine and Tissue Engineering-Cells and Biomaterials. InTechOpen; 2011.

66. Tang L, Thevenot P, Hu WJ. Surface chemistry influences implant biocompatibility. Curr Top Med Chem. 2008;8(4):270-280. doi:10.2174/156802608783790901

67. Hynes RO. Integrins: bidirectional, allosteric signaling machines. Cell. 2002;110(6):673-687. doi:10.1016/S0092-8674(02)00971-6

68. Cui Z-K, Kim S, Baljon JJ, Wu BM, Aghaloo T, Lee M. Microporous methacrylated glycol chitosan-montmorillonite nanocomposite hydrogel for bone tissue engineering. Nat Commun. 2019;10 (1):1-10. doi:10.1038/s41467-019-11511-3

69. Wong KH, Hynes MJ, Davis MA. Recent advances in nitrogen regulation: a comparison between Saccharomyces cerevisiae and filamentous fungi. Eukaryot Cell. 2008;7(6):917-925. doi:10.1128/ EC.00076-08
International Journal of Nanomedicine

\section{Publish your work in this journal}

The International Journal of Nanomedicine is an international, peerreviewed journal focusing on the application of nanotechnology in diagnostics, therapeutics, and drug delivery systems throughout the biomedical field. This journal is indexed on PubMed Central, MedLine, CAS, SciSearch ${ }^{\mathbb{}}$, Current Contents ${ }^{\mathbb{R}} /$ Clinical Medicine, $^{-}$

\section{Dovepress}

Journal Citation Reports/Science Edition, EMBase, Scopus and the Elsevier Bibliographic databases. The manuscript management system is completely online and includes a very quick and fair peer-review system, which is all easy to use. Visit http://www.dovepress.com/ testimonials.php to read real quotes from published authors. 